\title{
Magnetohydrodynamic simulations of gamma-ray burst jets: beyond the progenitor star
}

\author{
Alexander Tchekhovskoy ${ }^{\mathrm{a}}$, Ramesh Narayan ${ }^{\mathrm{a}}$, Jonathan C. McKinney ${ }^{\mathrm{b}}$
}

${ }^{a}$ Institute for Theory and Computation, Harvard-Smithsonian Center for Astrophysics, 60 Garden Street, Cambridge, MA 02138, USA
${ }^{b}$ Kavli Institute for Particle Astrophysics and Cosmology, Stanford University, P.O. Box 20450, Stanford, CA 94309, USA; Chandra Fellow

Abstract

'Achromatic breaks in afterglow light curves of gamma-ray bursts (GRBs) arise naturally if the product of the jet's Lorentz factor $\gamma$ and opening angle $\Theta_{j}$ satisfies $\gamma \Theta_{j} \gg 1$ at the onset of the afterglow phase, i.e., soon after the conclusion of the prompt emission. 'Magnetohydrodynamic (MHD) simulations of collimated GRB jets generally give $\gamma \Theta_{j} \lesssim 1$, suggesting that MHD models may be inconsistent with jet breaks. We work within the collapsar paradigm and use axisymmetric relativistic MHD simulations to explore the effect of a finite stellar envelope on the structure of the jet. Our idealized models treat the jet-envelope interface as a collimating rigid wall, which opens up outside the star to mimic loss of collimation. We find that the onset of deconfinement causes a burst of acceleration accompanied by a slight increase in the opening angle. In our fiducial model with a stellar radius equal to $10^{4.5}$ times that of the central compact object, the jet achieves an asymptotic Lorentz factor $\gamma \sim 500$ far outside the star and an asymptotic 'opening angle $\Theta_{j} \simeq 0.04 \mathrm{rad} \simeq 2^{\circ}$, giving $\gamma \Theta_{j} \sim 20$. These values are consistent with observations of typical long-duration GRBs, and explain the occurrence of jet breaks. We provide approximate analytic solutions that describe the numerical results well.

'Keywords: relativity, MHD, gamma rays: bursts, galaxies: jets, accretion, accretion disks, black hole physics

\section{Introduction}

Relativistic jets are ubiquitous features of many accreting black holes and neutron stars. They are found in systems 'spanning an enormous range of compact object mass: from $\mathrm{x}-$ ray binaries (XRBs) and gamma-ray bursts (GRBs) with neutron stars and stellar-mass black holes (BHs) of mass $M \sim$ $1.4-20 M_{\odot}$, to active galactic nuclei (AGN) with BHs of mass $M \sim 10^{6}-10^{10} M_{\odot}$. The physics of jet production appears to be robust and insensitive to the details of the central object.

Two characteristic features of relativistic jets are the following: (i) Jets are accelerated efficiently and achieve large Lorentz factors $\gamma$, ranging to above several hundred in the case of GRBs. (ii) Jets are strongly confined, with opening angles $\Theta_{j} \lesssim 0.1 \mathrm{rad}$. It is generally assumed that these two properties are related, though the exact relation is not well understood.

Long-duration GRBs (which we hereafter refer to simply as GRBs) are particularly interesting in this regard since we have -measurements of both $\gamma$ and $\Theta_{i}$ (Piran, 2005). Many GRBs have $\gamma \gtrsim 400$ (Lithwick \& Sari, 2001), with jet opening angles broadly distributed around a typical value $\Theta_{j} \sim 0.05 \mathrm{rad}$ (Zeh et al., 2006). These estimates imply that $\gamma \Theta_{j} \sim 10-30$.

Jet acceleration requires an energy source. In the absence of magnetic fields this source may be thermal energy injected into the jet by annihilating neutrinos from an accretion disk (Kohri et al., 2005; Chen \& Beloborodov, 2007; Kawanaka \& Mineshige, 2007; Zalamea \& Beloborodov,

Email addresses: atchekho@cfa.harvard.edu (Alexander Tchekhovskoy), rnarayan@cfa.harvard.edu (Ramesh Narayan), jmckinne@stanford.edu (Jonathan C. McKinney)
2009). Such thermally-driven jets accelerate because of expansion of the relativistically hot gas (e.g., Zhang et al., 2003, 2004; Morsony et al., 2007; Wang et al., 2008). However, self-consistent simulations including neutrino physics have not yet succeeded in launching relativistic jets (Nagataki et al. 2007; Takiwaki et al. 2009; Nagataki 2009; however, see Harikae et al. 2009). The inclusion of magnetic fields enables the extraction of rotational energy from the central compact object and this naturally produces relativistic jets Blandford \& Znajek 1977; McKinney \& Gammie 2004; McKinney 2006; Komissarov \& McKinney 2007; Tchekhovskoy et al. 2008, hereafter TMN08). In such models, acceleration is caused by the expansion of magnetic fields which have been twisted and amplified by the rotation of the central compact object (Begelman \& Li 1994; Komissarov et al. 2009, hereafter K09; Tchekhovskoy et al. 2009, hereafter TMN09). We concentrate on this magnetic acceleration mechanism in the present paper.

K09 argued, based on numerical simulations, that relativistic magnetohydrodynamic (MHD) jets confined by an external medium should have $\gamma \Theta_{j} \lesssim 1$. Their result appears to be in conflict with the GRB data summarized above. On the other hand, a completely unconfined split-monopole flow achieves larger opening angles and gives $\gamma \Theta_{j}>10$ (TMN09). How do these results relate to GRBs? According to the collapsar model Woosley, 1993; MacFadyen \& Woosley, 1999), GRB jets are produced by an accreting stellar-mass black hole at the center of a collapsing massive star. As the jet propagates out, it is collimated by the pressure of the shocked stellar envelope. However, once the jet emerges from the star it is effectively in vacuum, since the pressure of the external interstellar medium 
or stellar wind is far lower than the internal pressure in the jet. Thus a GRB jet corresponds to a hybrid scenario which involves initial confinement followed by free propagation. What are the asymptotic properties of such a jet far from the star? In particular, does $\gamma \Theta_{j}$ have a value of order unity, as in confined models (K09), or is it comparable to the values seen in unconfined splitmonopole models (TMN09)?

We have carried out high-resolution simulations that model the behavior of a relativistic magnetized jet emerging from stellar confinement. Our ideal MHD jets are confined out to a certain distance by a rigid wall of the appropriate shape to mimic the confining effect of the shocked stellar envelope (see \$2). We then allow the jets to be effectively unconfined beyond this distance. The simulations are meant to represent quasisteady GRB jets, i.e., we imagine that an initial (possibly nonrelativistic) precursor jet (e.g., Burrows et al., 2007) has already cleared the way for an ultrarelativistic magnetized jet which later powers the GRB. It is the latter jet that our simulations are meant to represent. The numerical setup is discussed in $\$ 2$ and the results are described in $\$ 3$. We conclude in $\$ 4$ with a discussion of the results in the context of GRBs. We work with Heaviside-Lorentzian units, neglect gravity, and set the speed of light and the compact object radius to unity: $c=r_{0}=1$. For a maximally-spinning $\mathrm{BH}$ with a characteristic mass $M=3 M_{\odot}$ (MacFadyen \& Woosley, 1999), our unit of length, in physical units, is $G M / c^{2} \approx 4.4 \times 10^{5} \mathrm{~cm}$.

\section{Numerical Method and Problem Setup}

We have performed our simulations using the relativistic MHD code HARM (Gammie et al., 2003; McKinney \& Gammie, 2004; McKinney, 2006), including recent improvements (Mignone \& McKinney 2007; Tchekhovskoy et al. 2007; TMN08; TMN09). We initialize the simulation with a purely poloidal field configuration in which the shape of each field line is given in polar coordinates by

$$
\theta(r) \propto r^{-v / 2}, \quad 0.5 \lesssim v \lesssim 1 .
$$

TMN08 showed that the above power-law shape corresponds to a power-law profile for the confining pressure:

$$
p \propto r^{-\alpha}, \quad \alpha=2(2-v) .
$$

We discuss our choice of $v$ in $\$ 3$ In the simulation, the jet shape (1) is maintained until a distance $r=r_{*}$, where $r_{*}$ represents the radius of the confining stellar envelope. Beyond $r_{*}$, we allow the field to decollimate.

In order to arrange for a smooth transition at $r_{*}$, we suitably choose the flux function $\Psi(r, \theta)$ of the initial magnetic field,

$$
\Psi(r, \theta)=(1-\cos \theta) / f^{2}(r),
$$

where we note that, by definition, $\Psi$ remains constant on field lines, and the total poloidal magnetic flux enclosed inside a polar angle $\theta$ at radius $r$ equals $2 \pi \Psi(r, \theta)$. The function $f(r)$ essentially sets the opening angle of the wall and we adjust this function so as to match our requirements. Inside the star, where the stellar envelope actively collimates the jet, we choose $f$ such that it decreases with distance as $f=\left(r / r_{\text {in }}\right)^{-v / 2}$, giving the collimating shape (11). Just outside the star, where pressure confinement ceases, we allow the wall to start decollimating. To model the deconfinement we choose $f(r)$ such that it reaches a minimum at $r \simeq r_{*}$ and then, within a radial distance $\Delta r=0.5 r_{*}$, smoothly transitions to a logarithmic increase with increasing $r$. The exact manner in which the wall opens up is unimportant as we discuss in $\$ 3$

The code uses internal coordinates $\left(x_{1}, x_{2}\right)$, which are uniformly sampled with $1536 \times 256$ grid cells. These are mapped to the physical coordinates $(r, \theta)$ via $r=2.1+\exp \left(x_{1}\right)$, and $x_{2}=\operatorname{sign}(\Psi)|\Psi|^{1 / 2}$. In the lateral $\theta$ direction, the computational domain extends from the polar axis $x_{2}=0$, where we apply antisymmetric boundary conditions, to $x_{2}=1$, where we place an impenetrable wall whose shape is determined by equation (3) with $\Psi=1$. In the radial direction, the computational domain extends from $r_{\text {in }}=3$ (three radii of the compact star 1 ) to $r_{\text {out }}=3 \times 10^{11}$. At $r=r_{\text {out }}$ we apply standard outflow boundary conditions, while at $r=r_{\text {in }}$ we apply jet injection boundary conditions (TMN09). In detail, at $r_{\text {in }}$ we set the jet magnetic flux by specifying the radial magnetic field component according to (3), $B_{r, 0}=r_{\text {in }}^{-2}$, and set the mass flux $j_{\mathrm{MA}}$ into the jet by specifying the plasma density $\rho_{0}=0.00132 B_{r, 0}^{2}\left(\approx 1.2 \times 10^{5} \mathrm{~g} \mathrm{~cm}^{-3}\right.$ in cgs units for $B_{r, 0}=10^{15} \mathrm{G}$ ) and poloidal 3-velocity $v_{0}=0.5$, both of which are independent of $\theta$.

We choose the rotational frequency of the central compact star to be $\Omega=0.25$, which corresponds to a maximally spinning BH, although we expect similar results for other values of $\Omega$ (Gammie et al., 2004; McKinney, 2005). We start the simulation with a non-rotating star and smoothly turn on the rotation. This generates a set of outgoing waves as the initial purely poloidal magnetic field develops a helical structure in response to the rotation. In the steady-state solution, which quickly establishes behind these waves and which we study below, energy flowing out of the compact star is the sum of (a) Poynting flux $j_{\mathrm{EM}}$, set by the rotation rate $\Omega$ and the field strength $B_{r, 0}$, and (b) kinetic energy flux $j_{\mathrm{KE}}$, set by $\rho_{0}$ and $v_{0}$. Poynting flux is converted to kinetic energy as plasma accelerates along a field line and their ratio defines the local magnetization of the field line, $\sigma=j_{\mathrm{EM}} / j_{\mathrm{KE}}$. As the field line accelerates, magnetization $\sigma$ decreases and Lorentz factor $\gamma$ increases in such a way that $\mu$, the ratio of the total energy flux $j_{\mathrm{EM}}+j_{\mathrm{KE}}$ to the mass flux $j_{\mathrm{MA}}$, is conserved, i.e.,

$$
\mu=\frac{j_{\mathrm{EM}}+j_{\mathrm{KE}}}{j_{\mathrm{MA}}}=\gamma(\sigma+1)=\text { constant along each field line, }
$$

where $j_{\mathrm{KE}}=\gamma j_{\mathrm{MA}}$. Since $\sigma \geq 0$, the value of $\mu$ determines the maximum possible Lorentz factor of the field line. The Poynting flux vanishes at the jet axis, so $\mu(\theta=0)=\gamma_{0}=\left(1-v_{0}^{2}\right)^{-1 / 2}$, and $\mu$ increases away from the axis roughly proportional to $\Psi$ (TMN09), reaching its maximum value, $\mu_{0} \approx 1150$ (for our choice of boundary conditions), at the jet boundary.

\footnotetext{
${ }^{1}$ It is a matter of convenience where we locate the inner surface at which we inject the jet. We have verified that the solution is insensitive to the choice if the flow is sub-Alfvénic at this surface.
} 


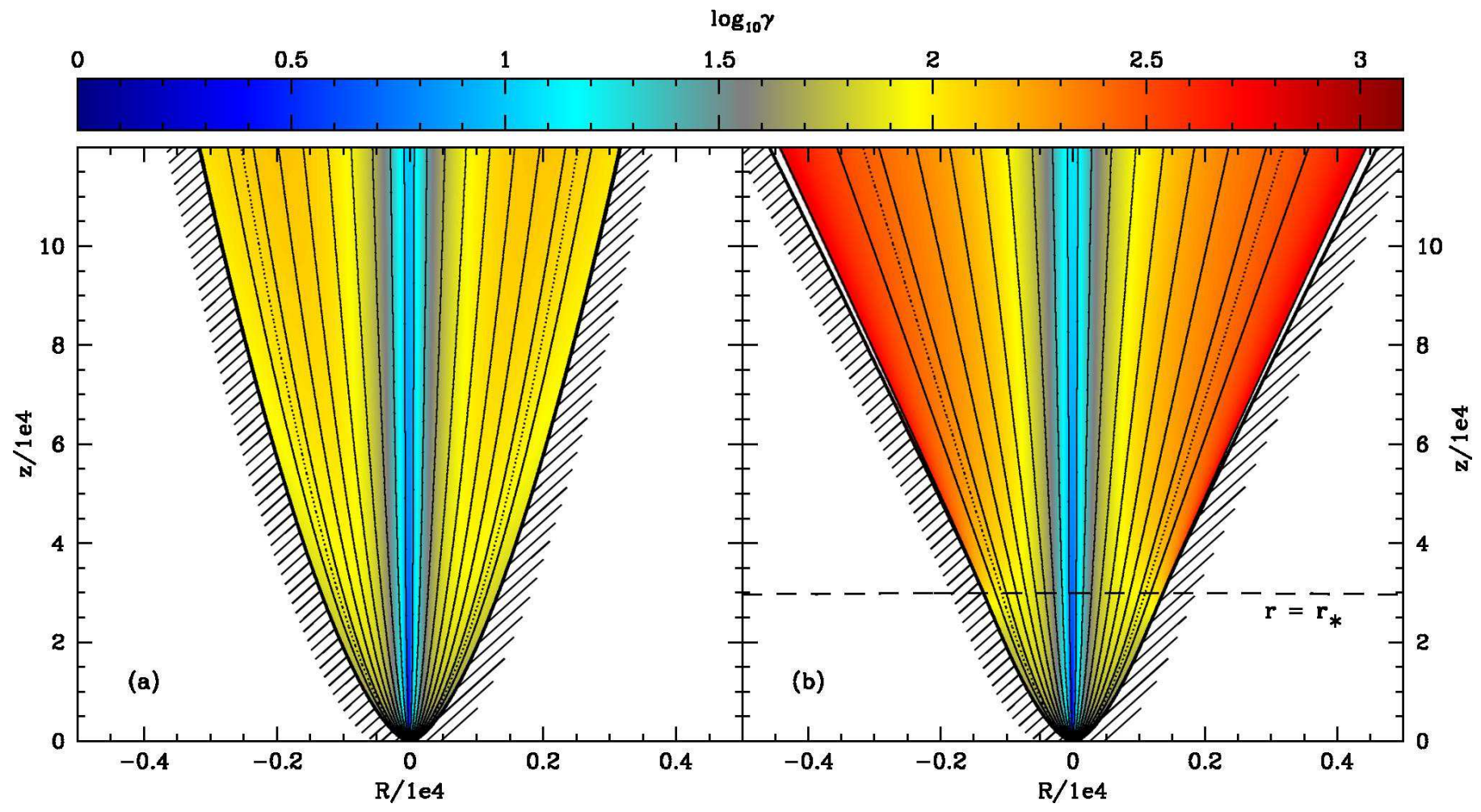

Figure 1: Meridional jet cross-section, showing color-coded logarithm of the Lorentz factor $\gamma$ overlaid with poloidal field lines (thin solid lines corresponding to $\left.\Psi^{1 / 2}=0.1,0.2, \ldots, 1\right)$. Thick solid lines show the position of the wall and dotted lines show the half-energy field line. [Panel a]: Jet confined continuously by a wall out to a large distance (model M $\infty$ ). [Panel b]: Jet confined by a collimating wall until $r=r_{*}=3 \times 10^{4}$ (model M4, where $r_{*}$ is shown by the horizontal dashed line), after which the wall opens up and the jet becomes deconfined. At this point, the jet separates from the wall and is surrounded effectively by vacuum. Once the jet becomes deconfined, $\gamma$ increases abruptly and the jet opening angle increases slightly.

In the context of GRBs, we are interested in a jet which asymptotically has a Lorentz factor of order a few hundred. Also, we assume that the jet energy goes into accelerated particles, and thereby to prompt GRB radiation, via an MHD shock. In order to have efficient conversion of jet energy into thermal energy at the shock, it is known that the jet fluid must achieve $\sigma \lesssim 1$ just prior to the shock (Kennel \& Coroniti, 1984). These conditions require $\mu \sim 10^{3}$ and motivate our choice of $\rho_{0} \sim 10^{-3}$. By choosing this value of $\mu$, we design our simulation such that, if the jet achieves a Lorentz factor of a few hundred, it will have $\sigma \lesssim 1$.

\section{Numerical Results}

We have run several simulations with the parameter $v$ set to $3 / 4$, and with different values of the confinement radius $r_{*}$ covering the likely range of progenitor star radii in GRBs. The choice $v=3 / 4$ is motivated by the work of McKinney \& Narayan (2007a b) and especially TMN08, who showed that this value corresponds to a confining pressure profile, $p \propto r^{-5 / 2}$ (eq. 2), which is reasonable for a jet-shocked stellar envelope, e.g., see hydrodynamic simulations of relativistic jets injected into GRB progenitor stars by Zhang et al. (2003). We have also run models with $v=2 / 3$ (i.e., $p \propto r^{-8 / 3}$ ), and the results are not qualitatively different.
As a baseline model, we first consider the case $r_{*} \rightarrow \infty$, i.e., a model in which the jet is continuously collimated by a wall out to an arbitrarily large distance. We refer to this as model M $\infty$. Figure 1 a shows a meridional cut through the steady-state solution we obtain at the end of the simulation. In this model, the jet $\gamma$ increases steadily and continuously and becomes of order a few hundred at large distance, while the jet opening angle decreases continuously with increasing distance.

Consider next a model with $r_{*}=3 \times 10^{4}\left(=1.3 \times 10^{10} \mathrm{~cm}\right.$ for a $3 M_{\odot}$ black hole). In this model, which we refer to as model M4, the wall collimates exactly as in model Mo until $r \sim r_{*}$, but the wall then smoothly opens up (as described by eq. 3), allowing the jet to decollimate. The steady state solution is shown in Figure 1 $1 \mathrm{~b}$. Just beyond $r=r_{*}$, soon after the jet loses confinement, we see that (a) the Lorentz factor $\gamma$ increases abruptly by a factor $\sim 10$ and (b) the opening angle of the jet $\Theta_{j}$ shows a slight increase and then "freezes out," hardly changing thereafter.

Figure 2 shows the radial dependence of various quantities. In addition to the models M $\infty$ and M4 already discussed, we consider two additional models: model M3 with $r_{*}=3 \times 10^{3}$ $\left(1.3 \times 10^{9} \mathrm{~cm}\right)$ and model M5 with $r_{*}=3 \times 10^{5}\left(1.3 \times 10^{11} \mathrm{~cm}\right)$. For each model, we show results corresponding to the "halfpower" field line, i.e., the field line for which half the jet power is carried by field lines inside of this line and half by field lines 


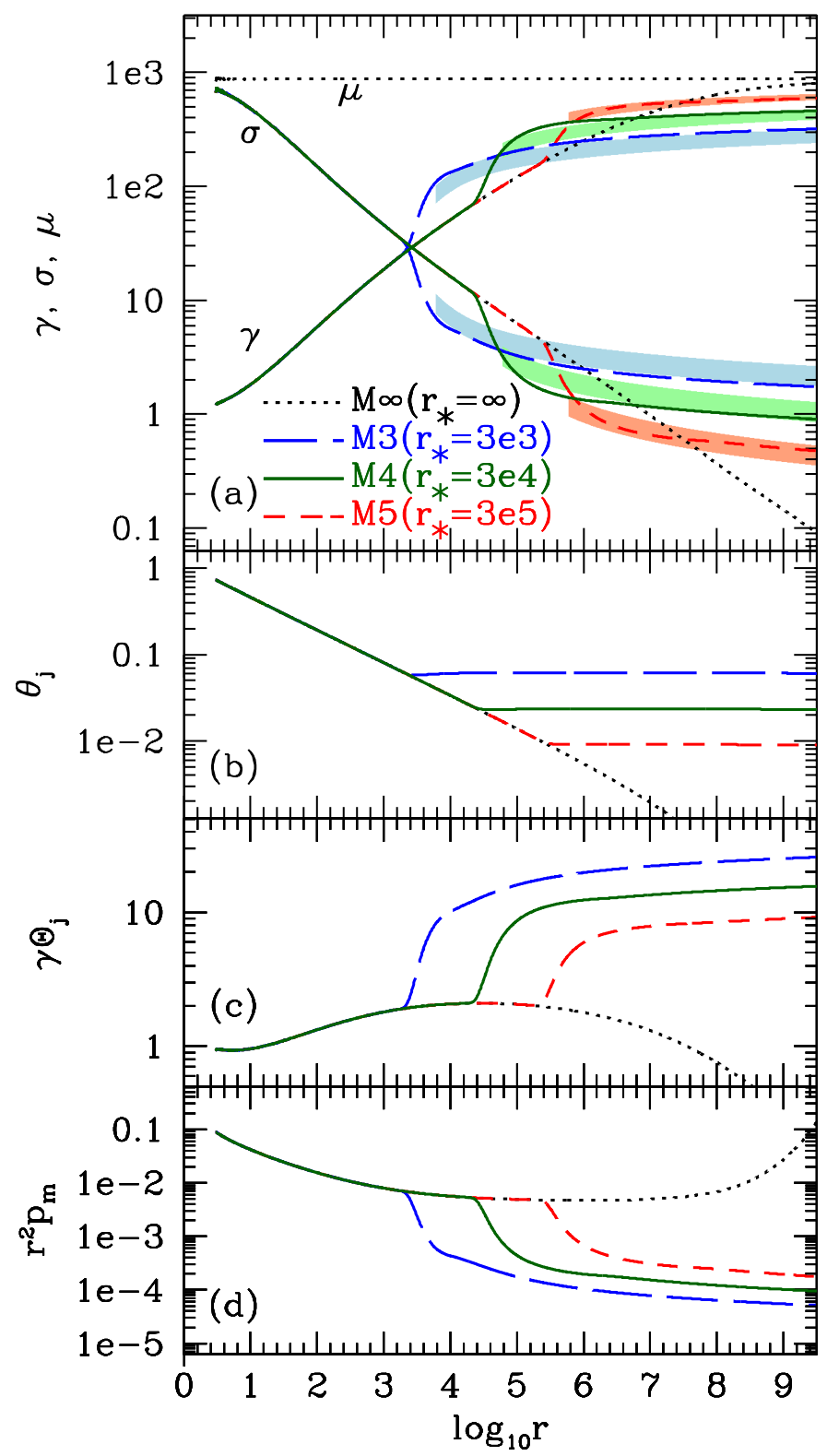

Figure 2: Dependence of various quantities along the half-energy field line for models M $\infty$, M3, M4, and M5. These models differ only in the jet confinement radius, i.e., the radius of the star $r_{*}$. [Panel a]: Lorentz factor $\gamma$, magnetization $\sigma$, and the total energy flux $\mu$. Model M $\infty$ shows continuous smooth acceleration, whereas the deconfined models M3-M5 show a "burst" of acceleration soon after the jet loses confinement. Shown with color stripes is the analytic solution for a fully unconfined jet (eq. 55. It reproduces our numerical solutions to within $20 \%$ (the width of the stripes). [Panel b]: Field line opening angle $\theta_{j}$. This decreases continuously while the jet is confined by the wall, but it hardly changes outside the star. [Panel c]: The product of the field line $\gamma$ and the full jet opening angle $\Theta_{j}$. In model $\mathrm{M} \infty$, we have $\gamma \Theta_{j} \lesssim 1$ at all $r$. However, in models M3-M5, $\gamma \Theta_{j}$ increases abruptly soon after the jet becomes unconfined and reaches values $\sim 10-30$, as appropriate for GRB jets. [Panel d]: Magnetic pressure $p_{\mathrm{m}}$. This decreases smoothly with increasing $r$ so long as the jet is confined $\left(r \lesssim r_{*}\right)$. However, once the wall opens up, $p_{\mathrm{m}}$ drops abruptly.

outside.

Figure 2 a shows as a function of $r$ the total energy flux $\mu$ (which is constant and the same for all models), the Lorentz factor $\gamma$, and the magnetization parameter $\sigma$. While model Mo

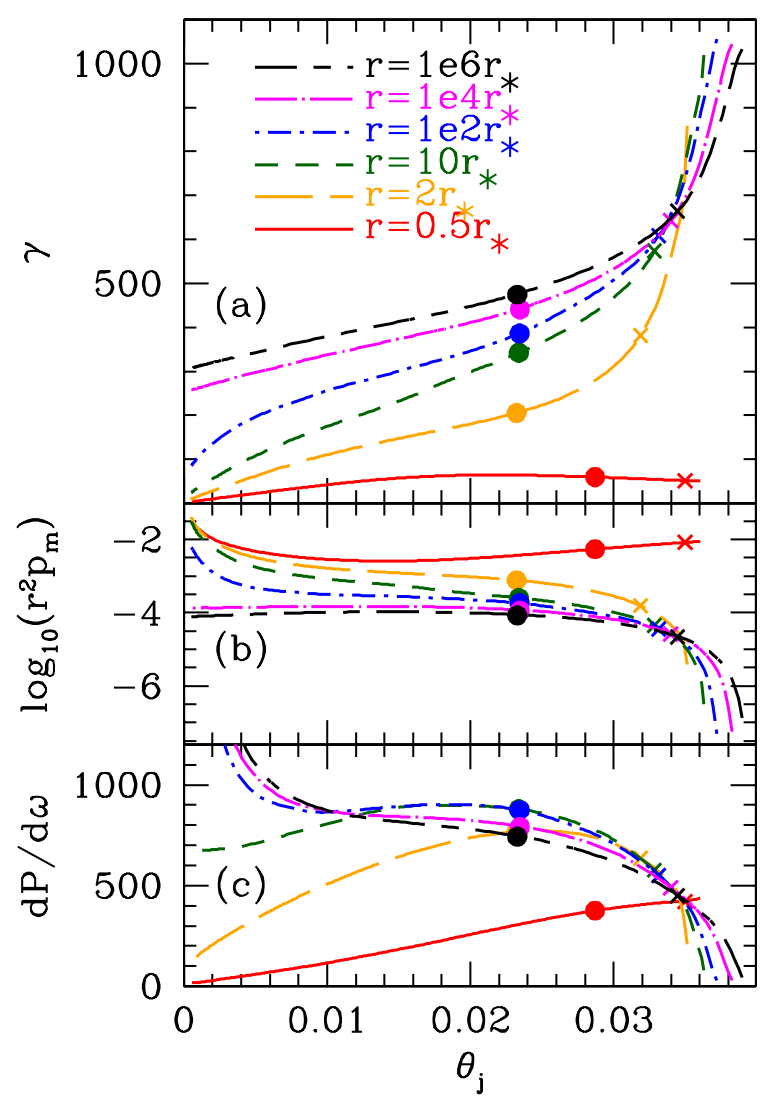

Figure 3: Angular distribution of $\gamma$ (panel a), magnetic pressure (panel b), and power output per unit solid angle (panel c) in our fiducial model M4 as a function of $\theta_{j}$, shown at various distances (see legend). Thick dots indicate the position of the half-energy field line. The collimation angle of this field line is nearly constant, $\theta_{j} \approx 0.024$, once the jet becomes deconfined. However, its Lorentz factor increases dramatically. Crosses indicate the full jet opening angle $\Theta_{j}(\$ 3$. Once the jet leaves the star, magnetic pressure and power output along the outermost field lines drop essentially to zero. Thus, outside the star, the jet is surrounded by a vacuum.

shows continuous smooth acceleration out to large radius, each of the three deconfined models, M3, M4 and M5, exhibits an abrupt increase in $\gamma$ soon after the jet loses confinement, with most of the acceleration occurring between about $r_{*}$ and $2 r_{*}$.

Figure $2 \mathrm{~b}$ shows the dependence of the angle $\theta_{j}$ between the poloidal component of the half-power field line and the jet axis. We use $\theta_{j}$ for the opening angle of a given field line and $\Theta_{j}$ for the opening angle of the entire jet; for the latter we use the field line that contains $90 \%$ of the jet's power output. Figure2 2 shows that, so long as the jet is confined $\left(r \lesssim r_{*}\right), \theta_{j}$ decreases with distance as $r^{-3 / 8}$ (eq. 1 with $v=3 / 4$ ). However, once the jet crosses $r_{*}$, the opening angle of the field line freezes and remains nearly constant out to arbitrarily large distance. Therefore, it is the opening angle of the wall (eq.3) as evaluated at $r=r_{*}$ that determines the asymptotic value of the jet opening angle. We note that the opening angles of our jets are similar to those found in hydrodynamic simulations of GRB jets (e.g., Zhang et al., 2003). This is perhaps not surprising since we calibrate the shape of the confining wall in our models by the shape of the jet boundary in the hydrodynamic simulations. 
After the burst of acceleration between $r_{*}$ and $2 r_{*}$, our deconfined jets subsequently accelerate very weakly. This is similar to unconfined magnetically-accelerated outflows which are known to accelerate only logarithmically at large distance (Beskin et al. 1998, TMN09). This is in contrast to thermally-accelerated outflows which continue to accelerate efficiently until they reach their maximum Lorentz factor (e.g., Zhang et al., 2003; Morsony et al., 2007).

Once an initially confined jet is allowed to become deconfined, its structure rearranges and begins to resemble a fully unconfined jet. Figure 2 a shows as colored stripes the approximate analytic solution for unconfined outflows obtained by TMN09:

$$
\gamma=2\left(\frac{\mu-\gamma}{\theta_{j}^{2}} \log \frac{\Omega R}{\gamma_{c}}\right)^{1 / 3}, \quad \gamma_{c}=\left(\frac{\mu}{\sin ^{2} \theta_{j}}\right)^{1 / 3} .
$$

Here $R=r \sin \theta_{j}$ is the cylindrical radius, $\log$ is the natural logarithm, and $\theta_{j}=(1-v / 2)(2 \Psi)^{1 / 2}\left(r_{*} / r_{\text {in }}\right)^{-v / 2}$ is the angle of the half-power field line, evaluated at the stellar surface (equation (3) with $\Psi \approx 0.7$ and $\left.f=\left(r_{*} / r_{\text {in }}\right)^{-v / 2}\right)$. The agreement of the analytical solution with our numerical results is better than 20\%. This agreement suggests that, once deconfined, our jets rapidly relax to the equivalent unconfined monopole solution for the same angle $\theta_{j}$ by converting their excess magnetic energy into kinetic energy. There is, however, an important difference. In a pure monopole solution, most of the jet energy flows out in the equatorial region, whereas in our jet models, because of the prior collimation, the energy flow is confined to within $0 \leq \theta_{j} \leq \Theta_{j}$.

Figure 2r shows the radial variation of the product $\gamma \Theta_{j}$, where $\Theta_{j}$ is the angle of the jet boundary (the field line with $\Psi=0.95$, which encloses $90 \%$ of the jet's total power). For the continuously collimated jet in model Mo (dotted line), $\gamma \Theta_{j}$ is of order unity throughout the acceleration zone, as noted by K09, decreasing to a much smaller value at large distance. The unconfined jets in M3, M4 and M5, however, behave very differently. Once these jets become unconfined, $\gamma \Theta_{j}$ increases rapidly from $\simeq 1$ to $\sim 10-30$. An angular power-densityweighted average of $\gamma \theta_{j}$ gives values within $30 \%$ of this estimate, which shows that the details of how we select $\gamma$ and $\Theta_{j}$ are unimportant. (A jet break is expected only if a large fraction of the power has $\gamma \theta \gg 1$; the power-density weighting accounts for this.)

What determines the value of $\gamma \Theta_{j}$ for a jet? To find this out, let us rewrite equation (5) in terms of magnetization, using equation (4):

$$
\gamma \Theta_{j}=2^{3 / 2}\left(\Theta_{j} / \theta_{j}\right) \sigma^{1 / 2} \log ^{1 / 2} \frac{\Omega R}{\gamma_{c}} \simeq 15 \sigma^{1 / 2},
$$

where in the last equality we assumed characteristic values for jet parameters in model M4 $\left(r=10^{9}, \theta_{j}=0.02, \Omega=0.25\right.$, $\left.\mu=10^{3}, \Theta_{j} / \theta_{j}=1.5\right)$. From this equation we clearly see that jets with subdominant magnetic fields at large distance, $\sigma \lesssim 1$, are limited to $\gamma \Theta_{j} \lesssim 15$.

We note that higher values of $\gamma \Theta_{j}$ correspond to deconfined models with smaller values of $r_{*}$. From the simulation results shown in Figure 2k, we obtain a scaling

$$
\gamma \Theta_{j} \propto r_{*}^{-0.22}
$$

We can use the analytic solution of fully unconfined jets to understand this trend. As we show in the Appendix, for models M3-M5, which are asymptotically roughly in equipartition ( $\gamma \approx \mu / 2$ at large distance), the analytic solution (5) gives:

$$
\gamma \simeq \mu / 2: \quad \Theta_{j} \propto r_{*}^{-v / 2}, \quad \gamma \propto \mu^{1 / 2} r_{*}^{v / 4}, \quad \gamma \Theta_{j} \propto \mu^{1 / 2} r_{*}^{-v / 4} .
$$

This implies $\gamma \Theta_{j} \propto r_{*}^{-0.19}$ for $v=3 / 4$, in good agreement with the numerical scaling (7). In the limit of high magnetization, i.e., $\sigma \gg 1$ and $\gamma \ll \mu$, we obtain instead (see the Appendix)

$$
\gamma \ll \mu: \quad \Theta_{j} \propto r_{*}^{-v / 2}, \quad \gamma \propto \mu^{1 / 3} r_{*}^{\nu / 3}, \quad \gamma \Theta_{j} \propto \mu^{1 / 3} r_{*}^{-v / 6} .
$$

Therefore, smaller values of stellar radius $r_{*}$ and larger values of $\mu$ give larger values of $\gamma \Theta_{j}$. However, in this limit, the asymptotic value of $\sigma$ will be $\gg 1$. Such jets cannot efficiently convert their Poynting energy flux into particle thermal energy via an MHD shock and therefore it is not clear if they will have sufficient energy in accelerated electrons to produce the prompt $\gamma$-ray emission observed in GRBs (see end of \$2). Other particle acceleration mechanisms, e.g., magnetic reconnection or plasma instabilities (e.g., Beloborodov, 2009), or different mechanisms, e.g., inverse Compton scattering of thermal photons (e.g., Broderick, 2005), may be able to circumvent this limit.

Finally, Figure 2 $\mathrm{d}$ shows the profile of magnetic pressure along the half-power field line. In the confined region of the flow, the pressure varies roughly as $r^{-5 / 2}$, leveling off to $\sim r^{-2}$ at larger distance 2 However, as the jet emerges from confinement, poloidal field lines rarefy laterally and the pressure decreases by over a factor of 10 (see models M3-M5). The drop in pressure creates a strong longitudinal pressure gradient which is the reason for the abrupt acceleration of the jet. This is an extreme example of the "magnetic nozzle" effect (Begelman \& Li 1994; TMN09) in action.

Figures 3 a,b,c show for model M4 the transverse profiles of $\gamma$, magnetic pressure $p_{\mathrm{m}}$, and power output per unit solid angle $d P / d \omega$ at various distances. We see that the abrupt acceleration and pressure drop experienced by the half-power field line (indicated by thick dots), described earlier in Figures 2a,d, is representative of most field lines. The outermost field lines near the edge of the jet $\left(\theta_{j}>\Theta_{j}\right)$ accelerate enormously during deconfinement but they contain little power $(\lesssim 10 \%)$ Lyubarsky 2009). Along these field lines the pressure drops essentially to zero once the jet emerges from the star, so these outer field lines are surrounded by vacuum.

We have included a fairly gentle opening up of the wall in the simulated models (e.g., see the shape of the wall in Fig.1p). However, because the jet moves ultrarelativistically and all signals are strongly beamed forward, even small changes in geometry are equivalent to nearly complete deconfinement, e.g.,

\footnotetext{
${ }^{2}$ The pressure profile in the slower magnetized sheath that surrounds the jet in a real system continues to follow the $p \propto r^{-5 / 2}$ dependence (TMN08).
} 
the jet surface in Fig. 1b clearly separates from the wall and the pressure at the jet surface (Fig. 3r) goes to zero. We have confirmed that our results are insensitive to the precise shape of the opening-up of the wall. Since the wall is causally disconnected from the flow, a more strongly diverging wall shape would not affect the results.

It should be noted that the numerical simulations described in this paper are extremely challenging since at high magnetization the equations of motion are very stiff and hard to solve. Additionally, it is a challenge to obtain accurate solutions that extend over 10 orders of magnitude in distance and in which ideal MHD conserved quantities are preserved along field lines to better than 15\% (K09; TMN09); our numerical models achieve this accuracy and are well-converged. To ensure that we have sufficient angular resolution near the pole and the wall, and sufficient radial resolution to resolve abrupt radial changes of quantities where the wall opens up, we have rerun model M4 with a locally 5 times higher angular resolution near the pole and near the wall, and also with a 5 times higher radial resolution between $0.5 r_{*}$ and $4 r_{*}$. We obtained less than $2 \%$ difference in asymptotic values of $\gamma$ and $\theta_{j}$ for most of the jet cross-section $\left(0.2<\theta_{j} / \Theta_{j}<1\right)$. After this work was posted on the archives, our results were confirmed by Komissarov et al. (2010) using a completely independent code.

\section{Discussion}

GRB afterglow radiation is produced when a relativistic jet ejected from a collapsar interacts with an external medium and decelerates. The afterglow phase typically starts at the end of the prompt gamma-ray emission, say at a time $t_{0} \sim 30 \mathrm{~s}$ in the observer frame. The Lorentz factor $\gamma$ and jet opening angle $\Theta_{j}$ discussed in the previous sections refer to the jet properties at this time. During the subsequent afterglow phase, the Lorentz factor $\gamma$ of the jet decreases with time. In the case of a uniform external medium and adiabatic evolution, $\gamma$ varies with expansion distance as $r^{-3 / 2}$ (Blandford \& McKee, 1976), which corresponds to a variation with observed time as $t^{-3 / 8}$ (e.g., Sari et al. 1998; Piran 2005). The opening angle of the jet $\Theta_{j}$, however, does not change. Therefore, during the afterglow phase of a GRB, the quantity $\gamma \Theta_{j}$ varies with observer time as

$$
\gamma \Theta_{j} \approx\left(t / t_{0}\right)^{-3 / 8}\left(\gamma \Theta_{j}\right)_{0}
$$

where the subscript 0 indicates values measured at the end of the prompt emission stage. When $\gamma \Theta_{j}$ falls below unity, there is an achromatic break in the afterglow light-curve and the observed flux falls more steeply with time (Rhoads, 1999; Sari et al., 1999). This "jet break" has been seen in a number of GRBs, typically about one to ten days after the initial prompt emission (Frail et al., 2001; Cenko et al., 2010). For these GRBs, according to equation (10), we require an initial $\left(\gamma \Theta_{j}\right)_{0} \sim(1 \text { day/30 s })^{3 / 8} \sim 20$ to explain the observations. This constraint has to be satisfied by any model of a GRB jet.

We find that both confinement by the collapsar's stellar envelope and free propagation outside the star are needed for an MHD jet to have (i) the required value of $\left(\gamma \Theta_{j}\right)_{0}$, (ii) the large power inferred in long-duration GRBs, and (iii) efficient conversion of electromagnetic to kinetic energy ( $\sigma \lesssim 1$ at large distance). Purely unconfined jets do give $\left(\gamma \Theta_{j}\right)_{0} \gg 1$ (TMN09). However, they have $\sigma \gg 1$ over most of their volume, which is undesirable, and they have too little power within the relevant opening angle where $\sigma \lesssim 1$. Purely confined jets are much better at focusing the power of the central engine into a collimated beam with $\sigma \lesssim 1$, but they give $\left(\gamma \Theta_{j}\right)_{0} \lesssim 1$. Only jets that are first confined and then deconfined can explain the most energetic long-duration GRBs with achromatic jet breaks. Such jets abruptly accelerate as soon as they emerge from the stellar envelope and develop a typical value of $\left(\gamma \Theta_{j}\right)_{0} \sim 20$, while at the same time achieving $\sigma \lesssim 1$ (Fig. 2). Remarkably, the stellar confinement radius we need to produce the observed value of $\left(\gamma \Theta_{j}\right)_{0}$ is $r_{*} \sim 10^{4-5}$ or a physical radius $\sim$ few $\times 10^{10} \mathrm{~cm}$, which agrees well with the estimated radii of GRB progenitor stars (e.g., Heger \& Langer, 2000).

Achromatic jet breaks have been observed in $20 \%$ of the Swift GRBs, but the actual number is much higher ( 50\%) since observations of most GRBs ceased before the expected jet break time (Kocevski \& Butler, 2008). As we have shown, current observations are putting stringent constraints on GRB models. If future observations by the Fermi Observatory routinely find $\gamma_{0}>10^{3}$ (Kumar \& Barniol Duran, 2009) and $\left(\Theta_{j}\right)_{0}>0.1$ (Cenko et al., 2010) for many GRBs, we would need to explain why these jets have $\left(\gamma \Theta_{j}\right)_{0}>100$. The numerical factor in equation (6) is a logarithmic factor and is unlikely to be larger than 10-20. Therefore, either we must accept that $\sigma \gg 1$, i.e., GRB jets are Poynting-dominated and somehow manage to convert a large fraction of their energy to prompt gamma-rays, or that MHD is not the appropriate framework for understanding GRB jets.

While we have focused primarily on GRB jets, our results also have some bearing on relativistic jets in XRBs and AGN. In order to produce collimated and efficient $(\sigma \lesssim 1)$ jets, the accretion disks in these systems must collimate the outflowing Poynting flux from the central BH. Strong winds are naturally present in advection-dominated accretion flows (ADAFs, Narayan \& Yi 1994, 1995; Narayan \& McClintock 2008), so one expects to see well-developed jets whenever XRBs and AGN are in the ADAF state. The exact properties of the emerging jet will presumably depend on the distance to which efficient wind-collimation operates.

We note that our simulations assume a fixed shape for the confining wall. However, as the GRB progresses and the jet disrupts the star, the opening angle of the jet is likely to increase. Our model does not take this effect into account. Also, our assumption of a rigid wall as the jet confining agent eliminates instabilities and mixing at the jet interface. These effects should be taken into account in future work, although the problem of a magnetized ultra-relativistic jet interacting with a surrounding progenitor star envelope is numerically extremely challenging and remains unsolved. Future work should also study jet stability to non-axisymmetric modes (Narayan et al., 2009), general relativistic effects of the central spinning BH (Tchekhovskoy et al., 2010), how GRB engines become threaded by the ordered magnetic fields needed for 
MHD jets (McKinney, 2006; McKinney \& Blandford, 2009), and should model radiation processes in order to make detailed comparisons with observed afterglow light curves and jet breaks.

\section{Acknowledgements}

We thank the anonymous referee for valuable comments on the manuscript. This work was supported in part by NASA grant NNX08AH32G (AT \& RN), NSF grant AST0805832 (AT \& RN), NASA Chandra Fellowship PF7-80048 (JCM), and by NSF through TeraGrid resources (Catlett et al., 2007) provided by the Louisiana Optical Network Initiative (www.loni.org) under grant number TG-AST080026N.

\section{References}

Begelman, M. C. \& Li, Z.-Y. 1994, ApJ, 426, 269

Beloborodov, A. M. 2009, ArXiv:0907.0732

Beskin, V. S., Kuznetsova, I. V., \& Rafikov, R. R. 1998, MNRAS, 299, 341

Blandford, R. D. \& McKee, C. F. 1976, Physics of Fluids, 19, 1130

Blandford, R. D. \& Znajek, R. L. 1977, MNRAS, 179, 433

Broderick, A. E. 2005, MNRAS, 361, 955

Burrows, A., Dessart, L., Livne, E., Ott, C. D., \& Murphy, J. 2007, ApJ, 664, 416

Catlett, C. et al. 2007, HPC and Grids in Action, Amsterdam

Cenko, S. B., Frail, D. A., Harrison, F. A., Kulkarni, S. R., Nakar, E., Chandra, P. C., Butler, N. R., Fox, D. B., Gal-Yam, A., Kasliwal, M. M., Kelemen, J., Moon, D., Ofek, E. O., Price, P. A., Rau, A., Soderberg, A. M., Teplitz, H. I., Werner, M. W., Bock, D., Bloom, J. S., Starr, D. A., Filippenko, A. V., Chevalier, R. A., Gehrels, N., Nousek, J. N., \& Piran, T. 2010, ApJ, 711, 641

Chen, W.-X. \& Beloborodov, A. M. 2007, ApJ, 657, 383

Frail, D. A. et al. 2001, ApJ, 562, L55

Gammie, C. F., McKinney, J. C., \& Tóth, G. 2003, ApJ, 589, 444

Gammie, C. F., Shapiro, S. L., \& McKinney, J. C. 2004, ApJ, 602, 312

Harikae, S., Kotake, K., \& Takiwaki, T. 2009, ArXiv:0912.2590

Heger, A. \& Langer, N. 2000, ApJ, 544, 1016

Kawanaka, N. \& Mineshige, S. 2007, ApJ, 662, 1156

Kennel, C. F. \& Coroniti, F. V. 1984, ApJ, 283, 694

Kocevski, D. \& Butler, N. 2008, ApJ, 680, 531

Kohri, K., Narayan, R., \& Piran, T. 2005, ApJ, 629, 341

Komissarov, S. S. \& McKinney, J. C. 2007, MNRAS, 377, L49

Komissarov, S. S., Vlahakis, N., \& Konigl, A. 2010, ArXiv:0912.0845

Komissarov, S. S., Vlahakis, N., Königl, A., \& Barkov, M. V. 2009, MNRAS, 394, 1182

Kumar, P. \& Barniol Duran, R. 2009, MNRAS, 400, L75

Lithwick, Y. \& Sari, R. 2001, ApJ, 555, 540

Lyubarsky, Y. 2009, ApJ, 698, 1570

MacFadyen, A. I. \& Woosley, S. E. 1999, ApJ, 524, 262

McKinney, J. C. 2005, ApJ, 630, L5

McKinney, J. C. 2006, MNRAS, 368, 1561

McKinney, J. C. \& Blandford, R. D. 2009, MNRAS, 394, L126

McKinney, J. C. \& Gammie, C. F. 2004, ApJ, 611, 977

McKinney, J. C. \& Narayan, R. 2007a, MNRAS, 375, 513

McKinney, J. C. \& Narayan, R. 2007b, MNRAS, 375, 531

Mignone, A. \& McKinney, J. C. 2007, MNRAS, 378, 1118

Morsony, B. J., Lazzati, D., \& Begelman, M. C. 2007, ApJ, 665, 569

Nagataki, S. 2009, ArXiv:0907.0561

Nagataki, S., Takahashi, R., Mizuta, A., \& Takiwaki, T. 2007, ApJ, 659, 512

Narayan, R., Li, J., \& Tchekhovskoy, A. 2009, ApJ, 697, 1681

Narayan, R. \& McClintock, J. E. 2008, New Astronomy Review, 51, 733

Narayan, R. \& Yi, I. 1994, ApJ, 428, L13

Narayan, R. \& Yi, I. 1995, ApJ, 444, 231

Piran, T. 2005, Reviews of Modern Physics, 76, 1143

Rhoads, J. E. 1999, ApJ, 525, 737

Sari, R., Piran, T., \& Halpern, J. P. 1999, ApJ, 519, L17
Sari, R., Piran, T., \& Narayan, R. 1998, ApJ, 497, L17

Takiwaki, T., Kotake, K., \& Sato, K. 2009, ApJ, 691, 1360

Tchekhovskoy, A., McKinney, J. C., \& Narayan, R. 2007, MNRAS, 379, 469

Tchekhovskoy, A., McKinney, J. C., \& Narayan, R. 2008, MNRAS, 388, 551

Tchekhovskoy, A., McKinney, J. C., \& Narayan, R. 2009, ApJ, 699, 1789

Tchekhovskoy, A., Narayan, R., \& McKinney, J. C. 2010, ApJ, 711, 50

Wang, P., Abel, T., \& Zhang, W. 2008, ApJS, 176, 467

Woosley, S. E. 1993, ApJ, 405, 273

Zalamea, I. \& Beloborodov, A. M. 2009, in American Institute of Physics Conference Series, Vol. 1133, American Institute of Physics Conference Series, ed. C. Meegan, C. Kouveliotou, \& N. Gehrels, 121-123

Zeh, A., Klose, S., \& Kann, D. A. 2006, ApJ, 637, 889

Zhang, W., Woosley, S. E., \& Heger, A. 2004, ApJ, 608, 365

Zhang, W., Woosley, S. E., \& MacFadyen, A. I. 2003, ApJ, 586, 356

\section{Appendix A. Analytic scaling of $\gamma \Theta_{j}$ in jets}

In this section, we analytically derive relations for $\gamma \Theta_{j}$. For this, we substitute $\Theta_{j} \propto r_{*}^{-v / 2}$ (see eq. 1) into equation (5) and neglect the logarithm:

$$
\frac{\gamma^{3}}{\mu-\gamma} \propto r_{*}^{v} .
$$

Now, we take a logarithm of both sides of equation A.1 and take a differential:

$$
3 \frac{d \gamma}{\gamma}-\frac{d \mu-d \gamma}{\mu-\gamma}=v \frac{d r}{r_{*}}
$$

Our models M3-M5 are asymptotically roughly in equipartition: $\gamma \simeq \mu / 2$ at large distance. Therefore, we substitute $\mu-\gamma=\gamma=\mu / 2$ in the result,

$$
4 \frac{d \gamma}{\gamma}-2 \frac{d \mu}{\mu}=v \frac{d r}{r_{*}}
$$

Integration of this equation gives:

$$
\gamma \simeq \mu / 2: \quad \Theta_{j} \propto r_{*}^{-v / 2}, \quad \gamma \propto \mu^{1 / 2} r_{*}^{v / 4}, \quad \gamma \Theta_{j} \propto \mu^{1 / 2} r_{*}^{-v / 4} .
$$

This implies $\gamma \Theta_{j} \propto r_{*}^{-0.19}$ for $v=3 / 4$, in good agreement with the numerical scaling (7). In the limit of high magnetization, i.e., $\sigma \gg 1$ and $\gamma \ll \mu$, we approximate $\mu-\gamma \approx \mu$ in equation (A.1) and obtain instead

$$
\gamma \ll \mu: \quad \Theta_{j} \propto r_{*}^{-v / 2}, \quad \gamma \propto \mu^{1 / 3} r_{*}^{v / 3}, \quad \gamma \Theta_{j} \propto \mu^{1 / 3} r_{*}^{-v / 6} .
$$

